\title{
Comparison of COVID-19 and common cold chemosensory dysfunction*
}

\author{
C. Huart ${ }^{1,2}$, C. Philpott ${ }^{3,4}$, I. Konstantinidis ${ }^{5}$, A. Altundag ${ }^{6,7}$, K.L. Whitcroft ${ }^{8}$, \\ E.M.C. Trecca ${ }^{9}$, M. Cassano ${ }^{9}$, Ph. Rombaux ${ }^{1,2}$, T. Hummel ${ }^{10}$ \\ 'Cliniques universitaires Saint-Luc, Brussels, Belgium \\ ${ }^{2}$ Institute of Neuroscience, Université catholique de Louvain, Brussels, Belgium \\ ${ }^{3}$ The Norfolk Smell \& Taste Clinic, James Paget University Hospital, Gorleston, UK \\ ${ }^{4}$ Norwich Medical School, University of East Anglia, Norwich, UK \\ 5 2nd Academic ORL Department, Papageorgiou Hospital, Aristotle University, Thessaloniki, Greece \\ ${ }^{6}$ Department of Otorhinolaryngology, Acibadem Taksim Hospital, Istanbul, Turkey \\ Department of Otorhinolaryngology, Biruni University Medicine Faculty, Istanbul, Turkey \\ ${ }^{8}$ UCL Ear Institute, University College London, London, UK \\ 9 Department of Otorhinolaryngology, University Hospital of Foggia, Foggia, Italy \\ o Smell \& Taste Clinic, Department of Otorhinolaryngology, TU Dresden, Dresden, Germany
}

Rhinology 58: 6, $623-625,2020$

https://doi.org/10.4193/Rhin20.251

*Received for publication:

May 22, 2020

Accepted: July 6, 2020

\section{To the Editor:}

Anosmia constitutes a prominent symptom of COVID-19. However, anosmia is also a common symptom of acute colds of various origins. In contrast to an acute cold, it appears from several questionnaire-based studies that in the context of COVID-19 infection, anosmia is the main rhinological symptom and is usually not associated with other rhinological symptoms such as rhinorrhoea or nasal obstruction ${ }^{(1)}$. Until now, no study has directly compared smell and taste function between COVID-19 patients and patients with other causes of upper respiratory tract infection (URTI) using valid and reliable psychophysical tests.

As it appears that COVID-19 behaves differently compared to other respiratory viruses with proposed mechanisms including cytokine storms ${ }^{(2)}$ and neurological tropism ${ }^{(3)}$, we hypothesized that patterns of chemosensory dysfunction differ between these two groups. If that was the case it might indicate different degrees of viral-associated damage at the levels of the olfactory epithelium, the olfactory bulb or more central olfactory-eloquent structures.

In this study, we aimed to objectively assess and compare olfactory and gustatory functions in 10 COVID-19 patients (PCR diagnosed, assessed on average 2 weeks after infection), 10 acute cold (AC) patients (assessed before the COVID-19 outbreak) and 10 healthy controls, matched for age and sex. Smell performance was assessed using the extended "Sniffin' Sticks" test battery ${ }^{(4)}$, while taste function was assessed using "taste strips" $^{\prime(5)}$. Receiver Operating Characteristic (ROC) curves were built to probe olfactory and gustatory scores in terms of their discrimination between COVID-19 and AC patients.
We found that global olfactory function as indicated by the combined threshold, discrimination and identification (TDI) scores, as well as the component discrimination (D) and identification (I) scores significantly differed between COVID-19 patients and controls $(p=0.002, p=0.001$ and $p<0.001$, respectively). Moreover, we found that identification scores of COVID-19 patients were significantly lower compared to $A C$ patients $(p=$ 0.015) (Table 1). TDI, D and I discriminated between COVID-19 and AC patients. Specifically, the I score showed excellent discrimination between these two populations, with a $100 \%$ sensitivity and $80 \%$ specificity for a cut-off value of $\leq 10$ (Table 2 ). We found that olfactory dysfunction had a favorable recovery in both groups, as all patients reported improvement in the weeks following the infectious event. Particularly, all COVID-19 patients all reported improvement at a mean follow-up of 18 days ( \pm 6 days), although only $30 \%$ reported complete recovery. Global and bitter gustatory performances of COVID-19 patients were also lower compared to controls $(p=0.025$ and $p=0.019$, respectively). In comparison to AC patients, we found that COVID-19 patients had worse global, sweet and bitter gustatory scores $(p=0.015, p=0.026$ and $p=0.001$, respectively) (Table 1$)$. These performances discriminated between AC and COVID-19 patients. The bitter stimulus showed an excellent discrimination performance with $90 \%$ sensitivity and $80 \%$ specificity (Table 2 ). Our results suggest different mechanisms involving the olfactory system in AC and COVID-19. Indeed, in the particular context of COVID-19 infection, the impairment of discrimination and identification performances may suggest the involvement of central olfactory structures. Supporting our results, Li et al. have suggested that SARS CoV-2 could have a neuroinvasive 
Table 1. Smell and taste performances (mean \pm standard deviation) of patients with acute cold (AC), COVID-19 infection (COV) and controls.

\begin{tabular}{|lccc}
\hline & AC group & COV group & Controls \\
\hline Age in years (mean \pm SD) & $48.8 \pm 18.0$ & $48.0 \pm 17.4$ & $48.0 \pm 17.4$ \\
\hline Smell & & & \\
TDI (/48) & $28.2 \pm 5.1$ & $21.1 \pm 6.1$ & $32.0 \pm 5.9$ \\
\hline T (/16) & $4.9 \pm 3.0$ & $4.8 \pm 2.2$ & $5.7 \pm 2.5$ \\
\hline D (/16) & $10.9 \pm 2.1$ & $7.9 \pm 2.5$ & $12.6 \pm 1.8$ \\
I (/16) & $12.4 \pm 1.1$ & $8.4 \pm 2.27$ & $13.6 \pm 2.5$ \\
\hline Taste & & & \\
Global (/16) & $12.4 \pm 2.7$ & $8.8 \pm 2.3$ & $12.3 \pm 2.45$ \\
Sweet (/4) & $3.7 \pm 0.5$ & $2.6 \pm 0.8$ & $2.9 \pm 1.2$ \\
Sour (/4) & $2.3 \pm 1.1$ & $1.9 \pm 0.6$ & $3.2 \pm 0.8$ \\
\hline Bitter (/4) & $3.6 \pm 0.7$ & $1.9 \pm 0.7$ & $3.2 \pm 0.8$ \\
\hline Salty (/4) & $2.8 \pm 1.1$ & $2.4 \pm 0.7$ & $3.3 \pm 0.9$ \\
\hline
\end{tabular}

Smell was assessed using the Sniffin' Sticks test (T: threshold, D: discrimination, I: Identification), while taste was assessed using the taste strips.

potential, based on neurologic signs developed by some patients, as well as SARS-CoV-2's similarities with SARS CoV, which has been previously reported to enter the brain, possibly via the olfactory sensory neurons ${ }^{(3)}$. Therefore, it is postulated that SARS-CoV-2 has a tropism for neurological structures, notably those associated with olfaction. In the present study, patients were assessed during the acute phase of their infection and we may expect that the majority of them will recover to normal olfactory function. However, it is likely that some patients will develop persistent post-infectious olfactory loss. Comparing chemosensory patterns of post-infectious olfactory loss due to COVID-19 or other URTIs could be very informative to understand mechanisms involved in both diseases.

Interestingly, we found that taste functions were significantly worse in COVID-19 patients compared to AC and controls. This result shows that taste disturbances reported by COVID patients appear to reflect an impairment in gustatory abilities, and not purely a retronasal olfactory dysfunction. Taste impairment may result from peripheral and/or central pathological involvement of gustatory pathways. At a peripheral level, it was shown in mice that inflammatory cytokines, resulting from viral infection, alter normal taste transduction and cell turnover in taste buds ${ }^{(6)}$. Due to the fact that COVID-19 infection is known to induce a cytokine storm, it can be proposed that this abnormal production of pro-inflammatory cytokines induces damage at taste receptors and alters their transduction. At a central level, similarly to what was found for SARS-CoV, it has been suggested that SARS-CoV-2 may involve the nucleus of the solitary tract, which is part of the taste pathways ${ }^{(3)}$.

Moreover, we found that sweet and bitter taste were shown to
Table 2. Discrimination performances of smell and taste tests to discriminate between acute cold and COVID-19 patients.

\begin{tabular}{|lcccc|}
\hline & AUC & Sensitivity & Specificity & p-value \\
\hline Smell & & & & \\
\hline TDI & 0.79 & 90 & 60 & $0.028^{*}$ \\
\hline T & 0.48 & 60 & 40 & 0.880 \\
\hline D & 0.80 & 90 & 70 & $0.023^{*}$ \\
\hline I & 0.95 & 100 & 80 & $0.001^{*}$ \\
\hline Taste & & & & \\
\hline Global & 0.86 & 90 & 70 & $0.006^{*}$ \\
\hline Sweet & 0.84 & 100 & 60 & $0.010^{*}$ \\
\hline Sour & 0.65 & 50 & 90 & 0.241 \\
\hline Salt & 0.64 & 70 & 50 & 0.290 \\
\hline Bitter & 0.93 & 90 & 80 & $0.001^{*}$ \\
\hline
\end{tabular}

be more impaired. Although it is not possible to generalise our results based on our small cohort, we think that this result is interesting since it is known that sweet and bitter receptors share some similarities. Indeed, these receptors belong to the group pf G-Protein coupled receptors (GPCRs), while sour and acid taste transduction are thought to use ion channels. Also, sweet and bitter taste receptors are known to play an important role in innate immunity ${ }^{(7)}$. Therefore, it should be further investigated as to whether genetic variation in sweet or bitter taste receptors might predispose to COVID infection or, conversely, whether SARS-CoV-2 infection modifies the expression or function of these receptors, either directly or through a "cytokine storm". Interestingly, the common feature of olfactory, sweet and bitter receptors is that they are GPCR. It was suggested that GPCR expressed at the level of lung epithelial cells may be involved in the mechanism of SARS-CoV-2 cells entry ${ }^{(8)}$. Hence, it might be postulated that GPCR expressed at the level of olfactory sensory neurons and taste receptor cells may also be possible targets of the virus, explaining the high rate of smell and taste involvement in these patients.

Practically, the finding that olfactory and taste tests are able to discriminate between COVID-19 and AC patients, with a high sensitivity and specificity suggest that such tests could be used as screening test to identify potentially COVID-19 positive patients. Although chemosensory tests could not replace formal diagnostic tools such as PCR, serology or chest CT, they could constitute an alternative when these tests are not available or when a quick screening is needed, particularly at the level of primary care or emergency department. However, further studies including a larger number of subjects are needed to confirm our results.

In conclusion, our results suggest that mechanisms of COVID-19 related olfactory dysfunction are different from those seen in an 
$\mathrm{AC}$ and may reflect, at least to some extent, a specific involvement at the level of central nervous system in some COVID-19 patients. In the future, studies to assess the prevalence of persistent anosmia and neuroanatomical changes on MRI correlated to chemosensory function, will be useful to understand these mechanisms.

\section{Conflict of interest}

Since 2018 Thomas Hummel did research together with and received funding from Sony, Stuttgart, Germany; Smell and Taste Lab, Geneva, Switzerland; Takasago, Paris, France; Bayer, Berlin, Germany; H. Lundbeck, Copenhagen, Denmark; Aspuraclip, Berlin, Germany. Carl Philpott is trustee of Fifth Sense.

\section{Authorship contribution}

All authors meet the ICMJE criteria:
1) Substantial contributions to the conception or design of the work; or the acquisition, analysis, or interpretation of data for the work; 2) Drafting the work or revising it critically for important intellectual content; 3) Final approval of the version to be published; 4) Agreement to be accountable for all aspects of the work in ensuring that questions related to the accuracy or integrity of any part of the work are appropriately investigated and resolved. $\mathrm{CH}$ and $\mathrm{ET}$ were specifically responsible for the data acquisition.

\section{Acknowledgement}

Caroline Huart is post-doctorate clinical master specialist funded by the FNRS. Carl Philpott received funding from NIHR (Programme Grant for Applied Research), Royal College of Surgeons Pump Priming Grants, Rosetrees Foundation, Bernice Bibby Trust and Sir Jules Thorne Trust.

\section{References}

1. Haehner A, Draf J, Draeger S, K. dW Hummel T. Predictive value of sudden olfactory loss in the diagnosis of COVID-19. ORL J Otorhinolaryngol Relat Spec. 2020;1-6

2. Pedersen SF, Ho YC. SARS-CoV-2: a storm is raging. J Clin Invest. 2020.

3. Li YC, Bai WZ, Hashikawa T. The neuroinvasive potential of SARS-CoV2 may play a role in the respiratory failure of COVID-19 patients. J Med Virol. 2020.

4. Hummel $T$, Sekinger $B$, Wolf SR, Pauli $E$ Kobal G. 'Sniffin' sticks': olfactory performance assessed by the combined testing of odor identification, odor discrimination and olfactory threshold. Chem Senses.
1997;22(1):39-52.

5. Landis BN, Welge-Luessen A, Brämerson A, Bende M, Mueller CA, Nordin S, et al. Normative values for the assessment of gustatory function using impregnated filter papers ("Taste Strips"). Arch ORL. 2008;256:242-8

6. Wang $H$, Zhou M, Brand J, Huang L. Inflammation and taste disorders: mechanisms in taste buds. Ann N Y Acad Sci. 2009;1170:596-603.

7. Workman AD, Palmer JN, Adappa ND, Cohen NA. The Role of Bitter and Sweet Taste Receptors in Upper Airway Immunity. Curr Allergy Asthma Rep. 2015;15(12):72.

8. Singh Y, Gupta G, Satija S, Pabreja K,
Chellappan DK, Dua K. COVID-19 transmission through host cell directed network of GPCR. Drug Dev Res. 2020.

Caroline Huart

Cliniques universitaires Saint-Luc

Avenue Hippocrate 10

Brussels

Belgium

Tel: + 3227646005

E-mail: caroline.huart@uclouvain.be 